\title{
SIC-Means: A Semi-fuzzy Approach for Clustering Data Streams Using C-Means
}

\author{
Amr Magdy and Mahmoud K. Bassiouny \\ Computer and Systems Engineering, Alexandria University, Egypt \\ amr.magdy@alex.edu.eg, eng.mkb@gmail.com
}

\begin{abstract}
In recent years, data streaming has gained a significant importance. Advances in both hardware devices and software technologies enable many applications to generate continuous flows of data. This increases the need to develop algorithms that are able to efficiently process data streams. Additionaly, real-time requirements and evolving nature of data streams make stream mining problems, including clustering, challenging research problems. Fuzzy solutions are proposed in the literature for clustering data streams. In this work, we propose a $\boldsymbol{S}$ oft $\boldsymbol{I}$ ncremental $\boldsymbol{C}$-Means variant to enhance the fuzzy approach performance. The experimental evaluation has shown better performance for our approach in terms of Xie-Beni index compared with the pure fuzzy approach with changing different factors that affect the clustering results. In addition, we have conducted a study to analyze the sensitivity of clustering results to the allowed fuzziness level and the size of data history used. This study has shown that different datasets behave differently with changing these factors. Dataset behavior is correlated with the separation between clusters of the dataset.
\end{abstract}

Keywords: Fuzz Clustering, Soft Clustering, Data Streams, C-Means, Clustering Data Streams.

\section{Introduction}

In recent years, the data stream model has gained a significant importance. Advances in both hardware devices and software technologies enable many applications to generate continuous flows of data. This has increased the sources of streaming information and thus the need to develop algorithms that are able to efficiently process data streams. These algorithms have to consider related scalability issues. Huge volumes of data with continuous and evolving nature of streaming data have introduced two main challenges; (i) infeasibility of storing the entire data (ii) and infeasibility of multiple passes processing. These challenges have motivated data research communities to provide appropriate solutions that are able to overcome these problems. Query processing over data streams [3, sketching of data streams [7] and mining problems [12] are the most studied problems in this context.

Data clustering problem is one of the most studied mining problems in the literature 17, 16. Data clustering techniques can be classified on different bases.

F. Schwenker and N. El Gayar (Eds.): ANNPR 2010, LNAI 5998, pp. 96 107, 2010.

(C) Springer-Verlag Berlin Heidelberg 2010 
One possible distinguishing characteristic is how the technique relates data points to different clusters. The literature presented three types: (i) hard clustering (ii) fuzzy clustering (iii) and soft clustering 24]. In hard clustering, each data point belongs to exactly one cluster. In fuzzy clustering each data point belongs to all clusters with different membership degrees. Soft clustering relaxes the absolute fuzziness constraint so it relates a data point to only a subset of clusters which are the most similar under some threshold of similarity. In this work we present a $\boldsymbol{S}$ oft $\boldsymbol{I}$ ncremental $\boldsymbol{C}$-Means variant for clustering streaming data; the SIC-Means approach.

\section{Related Work}

The literature of clustering data streams is fairly mature. Research work on dynamic clustering in incremental basis has started in late eighties in the context of information retrieval [5]. Adapting dynamic clustering techniques to work for data streams didn't show a great success due to the evolving nature of data streams. O'Callaghan et al. 22] proposed a k-median based clustering algorithm for streaming data. Their algorithm receives the streaming data in chunks, applies the clustering process and then frees the memory representing the clustering solution with weighted centroids. They obtain the weighted centroids of the entire stream received so far by applying the same algorithm to the centroids obtained from chunks. They showed that their algorithm outperformed BIRCH [31] in terms of sum of squared distance.

Aggarwal et al. 11 proposed an important framework for clustering data streams. They simply suggest to develop a two phase framework; an online phase and an offline phase. The online phase processes the continuous stream of data building sufficient statistics and collecting enough information to the offline phase to use in extracting data clusters. The importance of this framework is not only gained from the CluStream approach they proposed utilizing this framework, but also from being the basic idea of many important following research work in clustering data streams [23, 6, 18, 26, 27]. Park and Lee [23], Jia et al. [18] and Tu and Chen [26] use the online phase to build a data histogram. They then merge neighboring dense cells in clusters during the offline phase under different measures for density. DenStream algorithm 6] considers a different approache for both online and offline phases. They collect neighborhood density information during the online phase. They then use this information to build clusters in a similar way to conventional DBScan algorithm [11.

Many other approaches in the literature proposed solutions for clustering data streams under different assumptions and from different perspectives [13, 30, 21, 2, 8, 29, 4, 25, 19, 20, 9. However, all these algorithms proposed hard clustering solutions. Some applications generate data items that can belong to more than one cluster of data. Hore et al. [14] proposed a fuzzy incremental clustering solution for data streams based on C-means algorithm. This solution assumes that all data items belong to all clusters with different degrees of strength. Our research attempts to improve the pure fuzzy approach. We argue that assuming 
all data items to belong to all clusters may be misleading. Some data items are obviously far from some clusters. In general, we can assume that every data item can belong to one or more clusters under some threshold of similarity. We apply this soft clustering concept to the incremental clustering solution.

\section{SIC-Means}

Streaming data algorithms in the literature assume that data points arrive and get processed either in batches or one at a time. We consider the former paradigm where $n_{i}$ points arrive at time instant $t_{i}$. Our approach is an improvement to Hore's approach presented in [14] with applying the soft clustering concept [15]. We use a threshold $\alpha$ on allowed fuzziness so that after applying the fuzzy clustering process, a data point $p_{j}$ belongs to a cluster $c_{k}$ iff the corresponding degree of membership $u_{k j} \geq \frac{\alpha}{\text { number-of-clusters }}$.

The SIC-Means approach, which stands for Soft Incremental C-Means, takes three input parameters; number of clusters $c$, size of history used $h$ and fuzziness threshold $\alpha$. SIC-Means assigns a weight to each data point reflecting its importance. The effect of these weights needs to be incorporated in the objective function. Similar to [10, we define the fuzzy objective function to be minimized as follows:

$$
J_{m}(U, V)=\sum_{k=1}^{c} \sum_{j=1}^{n} u_{k j}^{m} w_{j}\left\|p_{j}-v_{k}\right\|
$$

Where

$U$ : fuzzy membership matrix

$V$ : cluster centers vector

$u_{k j}$ : the degree of membership of point $p_{j}$ in cluster $c_{k}$

$v_{k}$ : the center of cluster $c_{k}$

$w_{j}$ : the weight of point $p_{j}$

In our work, we consider $J_{1.5}(U, V)$ which has shown better experimental results in our experiments than other values of fuzzifier $m$. Furthermore, we use Euclidean distance as a dissimilarity measure. At any time instant $t_{i}$, SIC-Means clusters $N_{i}$ weighted points where:

$$
N_{i}=\min \left(n_{i}+c *(i-1), n_{i}+c * h\right), i \geq 1
$$

New data points are assigned unit weights while cluster centers of stage $i$ has weights $w_{k}$ 's where:

$$
w_{k}=\sum_{j=1}^{N_{i}} u_{k j}, 1 \leq k \leq c
$$

Algorithm 1 outlines the main steps of a single SIC-Means stage. At each stage, the clustering algorithm considers old centers of the last $h$ stages in addition to the newly received data points. If the current stage $i<h$, then we use all the available history up to the moment. After each time instant $t_{i}$, only $\min (c * i, c * h)$ weighted points are kept in memory representing the cluster centers for the last $h$ stages. 


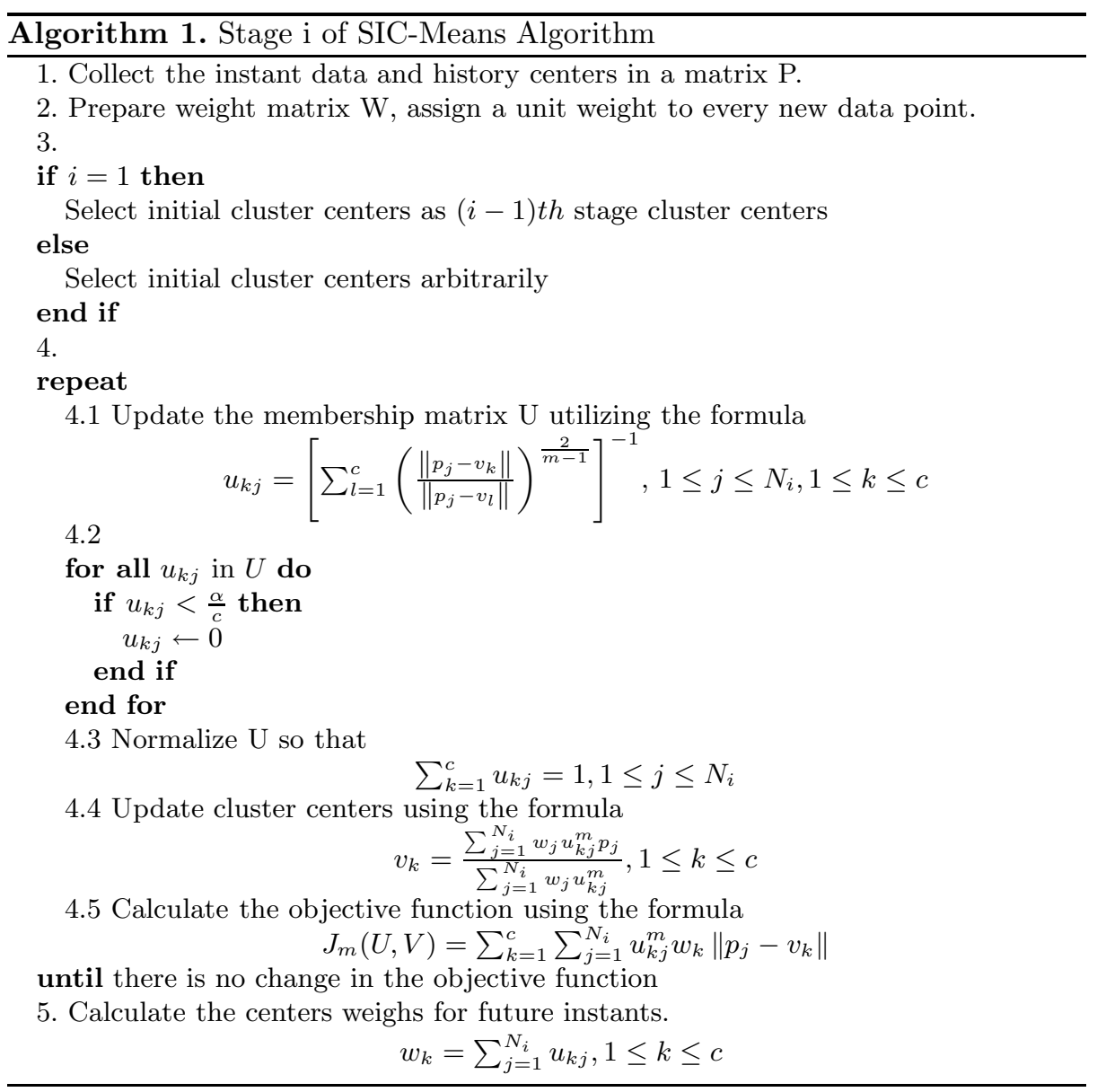

\section{Evaluation}

We have conducted a set of experiments to evaluate the performance of our approach. The experiments have shown an improvement, over using the pure fuzzy approach, under a standard evaluation scheme for fuzzy clustering algorithms. In this section, we first present the evaluation scheme we use, describe the evaluation datasets and then show the experimental results.

\subsection{Evaluation Scheme}

In our experiments, we use the popular Xie-Beni index [28] which is designed to internally validate fuzzy clustering solutions. XB index can be calculated using the following equation

$$
X B=\frac{1}{n} \frac{\sum_{\forall c_{k} \in C} \sum_{\forall p_{j} \in P} u_{k j}^{m}\left\|p_{j}-c_{k}\right\|}{\min _{\forall c_{l}, c_{k}}\left(\left\|c_{l}-c_{k}\right\|\right)}
$$


Where

$C:$ is set of all clusters

$P:$ is set of all data points

$m:$ is the fuzzifier

As discussed in 28], this index favors more compact and separate clusters. The lower the value of this index, the better the solution.

\subsection{Data}

In our evaluation, we use three artificial datasets; each of 100,000 2D points, to evaluate SIC-Means performance. The three datasets are drawn randomly from Gaussian distributions to form globular clusters. We have chosen these datasets for two main reasons. First, Hoer et al. 14] used one of these datasets to evaluate their algorithm. As we will introduce later in this section, we compare the performance of our new method relative to that of Hore et al. so it was appropriate to use the same dataset they used in evaluation. Second, a known limitation for C-means algorithm and its variants with Euclidean distance is to favor discovering globular clusters. In our research, we focus on showing the improvement of applying soft clustering concept to incremental fuzzy approaches. So we have chosen the datasets that serve our focus isolating the known C-means limitations.

The main difference between the three datasets is the separation between clusters. As shown in Figure 1, dataset DS1 clusters are strongly overlapped, dataset DS2 clusters are slightly overlapped and dataset DS3 clusters are well-separated. As soft clustering techniques is concerned with cutting off data points from far clusters, this variation in clusters separation should show the effectiveness of the technique.

\subsection{Experimental Results}

In our experiments, we have studied the effect of two major factors; fuzziness threshold $\alpha$ and size of history $h$. We have conducted our experiments using the true number of clusters studying the change in Xie-Beni index value with varying these factors. In these experiments, $\alpha=0$ represents the pure fuzzy approach which is Hore's approach. Figures 2- 4 show the results of these experiments.

As shown in Figure 2(a), XB index values on DS1 dataset for $0<\alpha \leq 0.6$ are lower compared to $\alpha=0$ for all history sizes (values of $h$ ). The upper bound of this range is extended to approach 0.8 at $\mathrm{h} \geq 2$. Figure 2(a) shows that history size greater than 2 does not provide a significant improvement for DS1 dataset; curves of history sizes 3 and 4 are approximately identical. These observations are supported by Figure 2(b) that shows obvious lower values of XB index for different $\alpha$ values with changing history size from 0 to 7 . In addition, excluding the pure fuzzy approach, at $\alpha=0$, in Figure 2(c) shows improvement with increasing history size up to $h=4$. Increasing history size after that degrades the performance. This can be explained by the evolving nature of data stream. According to the input order of the data points, more history weighted points 


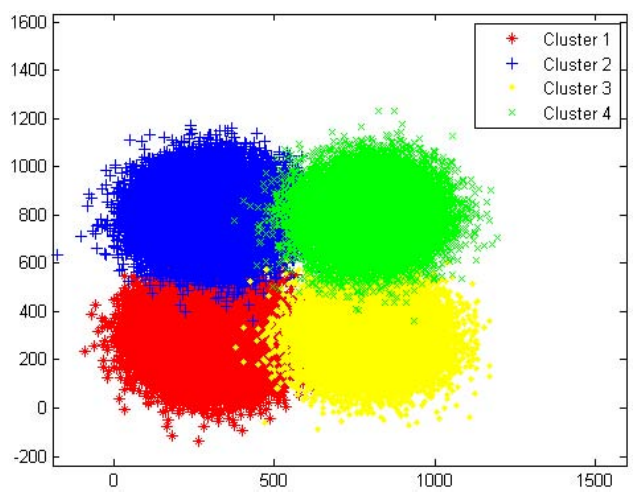

(a) Original distribution of dataset DS1

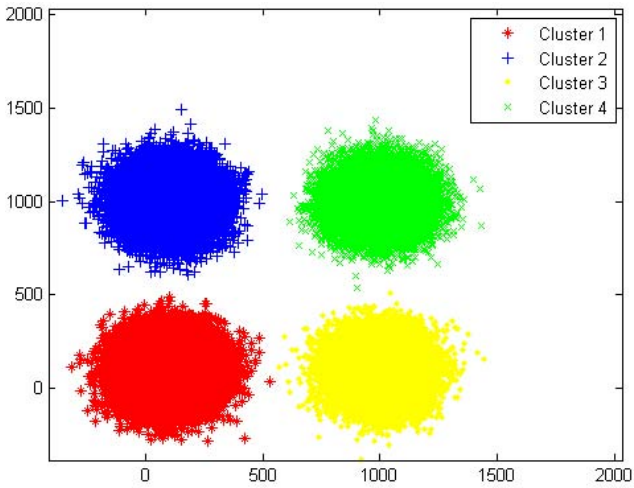

(b) Original distribution of dataset DS2

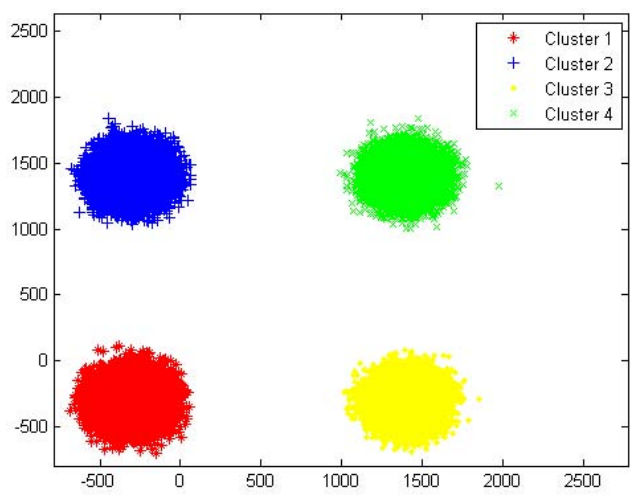

(c) Original distribution of dataset DS3

Fig. 1. Original distribution of evaluation datasets 


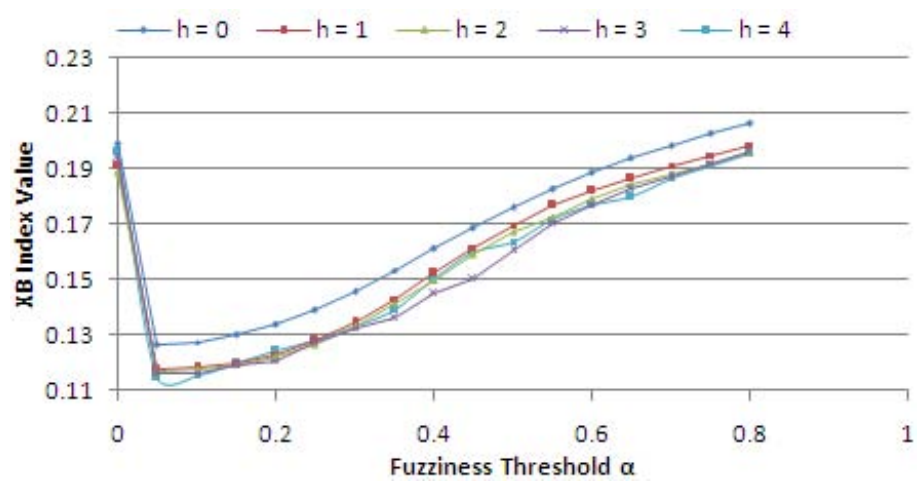

(a) SIC-Means performance for different history sizes

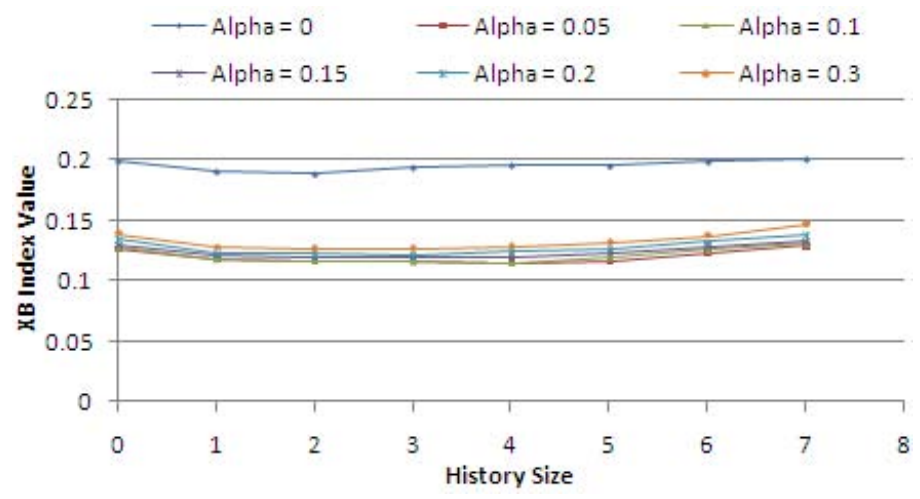

(b) SIC-Means performance for different fuzziness thresholds

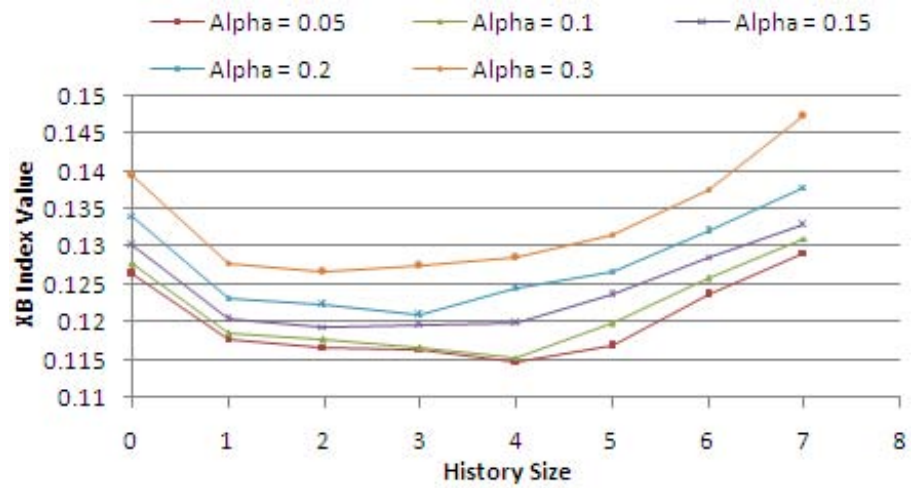

(c) SIC-Means performance for different fuzziness thresholds excluding pure fuzziness

Fig. 2. SIC-Means performance on DS1 dataset 


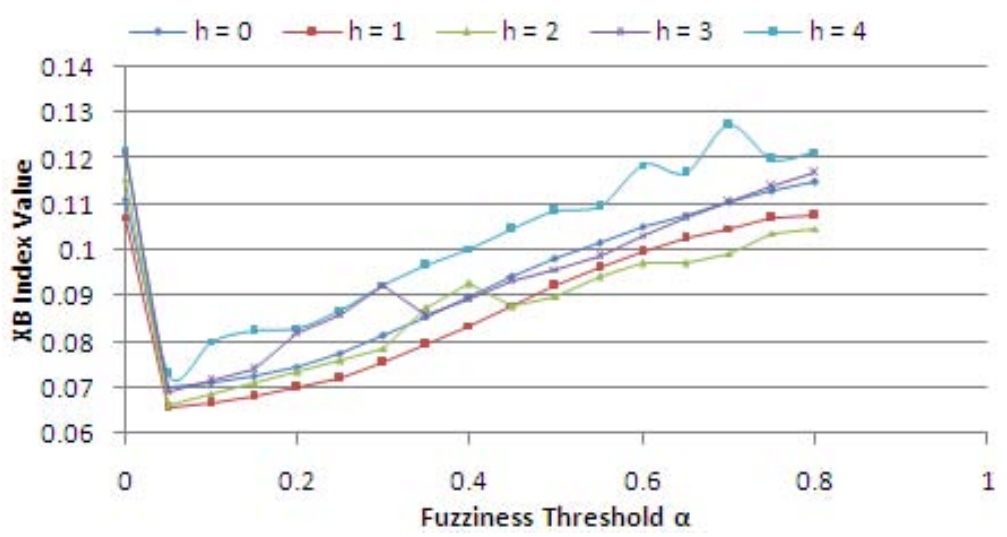

(a) SIC-Means performance for different history sizes

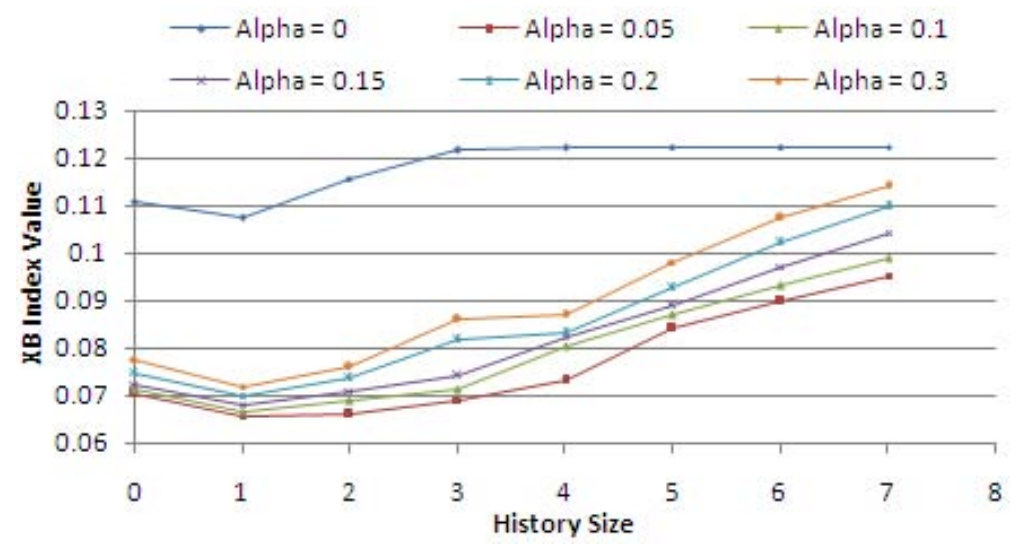

(b) SIC-Means performance for different fuzziness thresholds

Fig. 3. SIC-Means performance on DS2 dataset

can bias new points to the wrong clusters. However, it is obvious that considering more history does not always improve the overall performance.

Figure 3(a) shows similar observations on DS2 dataset. One obvious difference that $\mathrm{XB}$ index values are lower on DS2 dataset for wider range of $\alpha$ values, $0<\alpha \leq 0.8$ than the corresponding value at $\alpha=0$ for all history sizes $<4$. On the other hand, this shows an earlier performance degradation with increasing history size than DS1 dataset. These observations are supported by Figure 3(b)

Figure 4(a) can lead us to conclude that the more the clusters separated, the less the history needed. It shows almost no need to keep history data. Also this can be well observed in Figure 4(b). In almost all cases, the pure fuzzy approach is outperformed for all fuzziness thresholds and history sizes. 


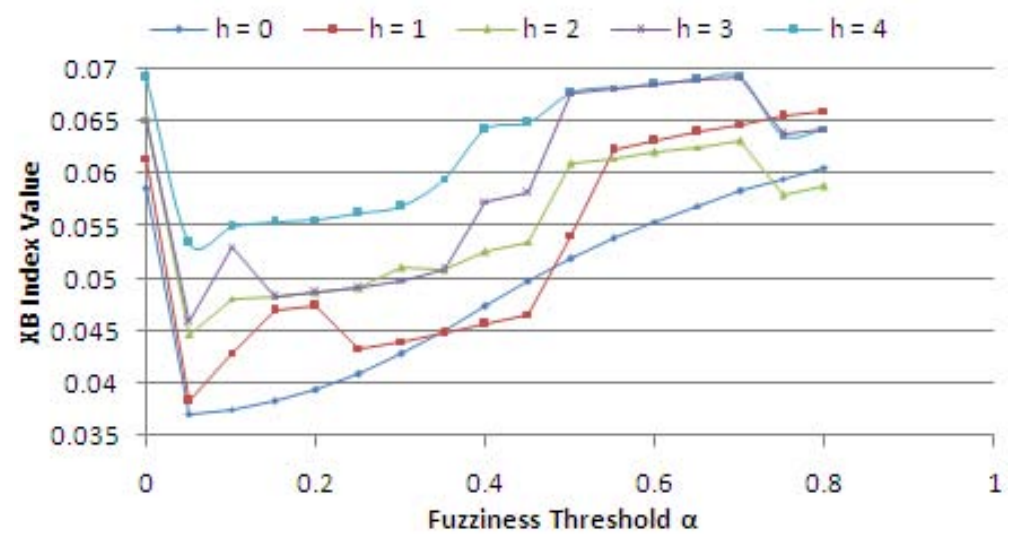

(a) SIC-Means performance for different history sizes

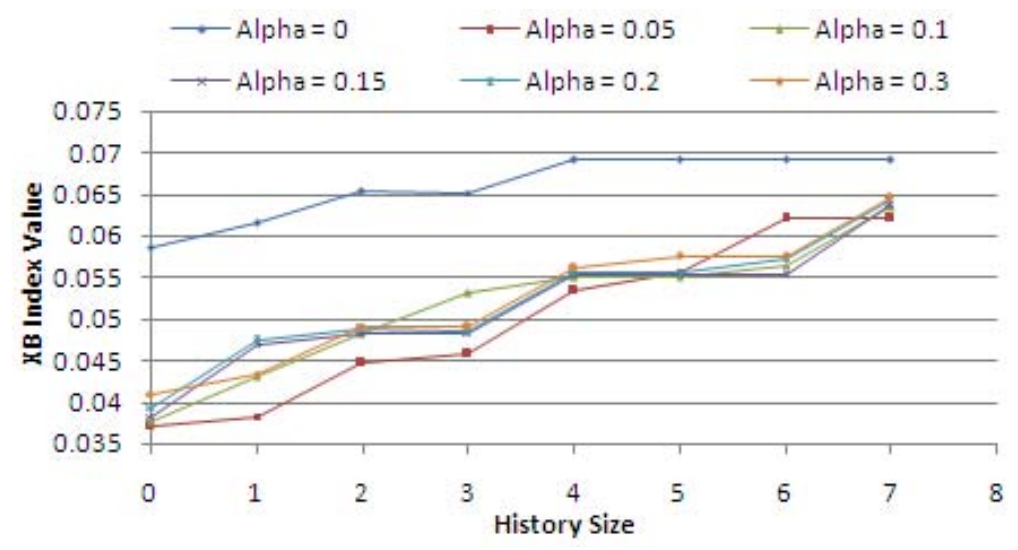

(b) SIC-Means performance for different fuzziness thresholds

Fig. 4. SIC-Means performance on DS3 dataset

\section{Conclusion}

In this paper, we introduced the combination of incremental and soft clustering concepts. We incrementally cluster batches of data points using weighted Cmeans variant. At any time instant, we consider a new batch of points with number of old batches of weighted points. These old points represent the cluster centers in earlier stages of the algorithm.

Combining incremental clustering with soft clustering has shown an ability to improve the fuzzy clustering results in terms of Xie-Beni index values. This improvement depends on different factors. From one hand, threshold for allowed 
fuzziness affect the performance. All thresholds of soft clustering used dominate the pure fuzzy clustering approach. However, considering more history does not always give better results. Evolving nature of data streams may bias new points to join the wrong clusters with increasing the history size above some value. History size value that separates performance improvement from performance degradation differs with changing the clusters separation. The better the clusters are separated, the lower history size needed. This property fits the limited memory constraint imposed by the data stream model.

\section{Discussion and Future Work}

In this work, we have shown the effectiveness of combining soft clustering technique with $\mathrm{C}$-means based incremental clustering technique. However, more evaluation techniques can be investigated. We may investigate building a fuzzy ground truth for evaluation datasets used. Existence of this ground truth will enable using external validation measures. Also, we may investigate how these clustering solutions tolerate errors in parameter selection; for example how they perform when clustering using wrong number of clusters. Other factors worth investigation are: sensitivity analysis of data input order and effect of using different dissimilarity measures.

Acknowledgment. The authors would like to thank Prof. Dr. M. A. Ismail for his support during Pattern Recognitions graduate course offered during the graduate studies in Computer and Systems Engineering Department, Alexandria University, Egypt. The basics of this work has been conducted as a part of the final project of this course.

\section{References}

1. Aggarwal, C.C., Han, J., Wang, J., Yu, P.S.: A framework for clustering evolving data streams. In: VLDB 2003: Proceedings of the 29th international conference on Very large data bases. VLDB Endowment, pp. 81-92 (2003)

2. Aggarwal, C.C., Han, J., Wang, J., Yu, P.S.: A framework for projected clustering of high dimensional data streams. In: VLDB 2004: Proceedings of the Thirtieth international conference on Very large data bases. VLDB Endowment, pp. 852-863 (2004)

3. Babcock, B., Babu, S., Datar, M., Motwani, R., Widom, J.: Models and issues in data stream systems. In: PODS 2002: Proceedings of the twenty-first ACM SIGMOD-SIGACT-SIGART symposium on Principles of database systems, pp. 1-16. ACM, New York (2002)

4. Beringer, J., Hüllermeier, E.: Online clustering of parallel data streams. Data Knowledge Engineering 58(2), 180-204 (2006)

5. Can, F., Ozkarahan, E.: A dynamic cluster maintenance system for information retrieval. In: SIGIR 1987: Proceedings of the 10th annual international ACM SIGIR conference on Research and development in information retrieval, pp. 123-131. ACM, New York (1987) 
6. Cao, F., Ester, M., Qian, W., Zhou, A.: Density-based clustering over an evolving data stream with noise. In: Proceedings Sixth SIAM Intl. Conference Data Mining (2006)

7. Cormode, G., Garofalakis, M.: Sketching probabilistic data streams. In: SIGMOD 2007: Proceedings of the 2007 ACM SIGMOD international conference on Management of data, pp. 281-292. ACM, New York (2007)

8. Dai, B.-R., Huang, J.-W., Yeh, M.-Y., Chen, M.-S.: Clustering on demand for multiple data streams. In: ICDM 2004. Fourth IEEE International Conference on Data Mining, November 2004, pp. 367-370 (2004)

9. Dang, X.H., Lee, V.C., Ng, W.K., Ong, K.L.: Incremental and adaptive clustering stream data over sliding window. In: Bhowmick, S.S., Küng, J., Wagner, R. (eds.) DEXA 2009. LNCS, vol. 5690, pp. 660-674. Springer, Heidelberg (2009)

10. Eschrich, S., Ke, J., Hall, L., Goldgof, D.: Fast accurate fuzzy clustering through data reduction. IEEE Transactions on Fuzzy Systems 11(2), 262-270 (2003)

11. Ester, M., Kriegel, H., Sander, J., Xu, X.: A density-based algorithm for discovering clusters in large spatial databases with noise. In: Proc. 2nd Int. Conf. on Knowledge Discovery and Data Mining, Portland, OR, pp. 226-231. AAAI Press, Menlo Park (1996)

12. Gaber, M.M., Zaslavsky, A., Krishnaswamy, S.: Mining data streams: a review. SIGMOD Record 34(2), 18-26 (2005)

13. Guha, S., Meyerson, A., Mishra, N., Motwani, R., O'Callaghan, L.: Clustering data streams: Theory and practice. IEEE Transactions on Knowledge and Data Engineering 15(3), 515-528 (2003)

14. Hore, P., Hall, L., Goldgof, D.: A fuzzy c means variant for clustering evolving data streams. In: ISIC. IEEE International Conference on Systems, Man and Cybernetics, October 2007, pp. 360-365 (2007)

15. Ismail, M.: Soft clustering: algorithms and validity of solutions. Fuzzy Computing, 445-472 (1988)

16. Jain, A.: Data Clustering: 50 Years Beyond K-Means. Pattern Recognition Letters (2009)

17. Jain, A.K., Murty, M.N., Flynn, P.J.: Data clustering: a review. ACM Computing Surveys 31(3), 264-323 (1999)

18. Jia, C., Tan, C., Yong, A.: A grid and density-based clustering algorithm for processing data stream. In: WGEC 2008: Proceedings of the 2008 Second International Conference on Genetic and Evolutionary Computing, pp. 517-521. IEEE Computer Society, Los Alamitos (2008)

19. Liu, Y., Cai, J., Yin, J., Fu, A.: Clustering text data streams. Journal of Computer Science and Technology 23(1), 112-128 (2008)

20. Lühr, S., Lazarescu, M.: Connectivity based stream clustering using localised density exemplars. In: Washio, T., Suzuki, E., Ting, K.M., Inokuchi, A. (eds.) PAKDD 2008. LNCS (LNAI), vol. 5012, pp. 662-672. Springer, Heidelberg (2008)

21. Nasraoui, O., Uribe, C., Coronel, C., Gonzalez, F.: Tecno-streams: tracking evolving clusters in noisy data streams with a scalable immune system learning model. In: ICDM 2003. Third IEEE International Conference on Data Mining, November 2003, pp. 235-242 (2003)

22. Callaghan, L.O., Mishra, N., Meyerson, A., Guha, S., Motwani, R.: Streamingdata algorithms for high-quality clustering. In: Proceedings of the International Conference on Data Engineering, pp. 685-696. IEEE Computer Society Press, Los Alamitos (2002)

23. Park, N.H., Lee, W.S.: Statistical grid-based clustering over data streams. SIGMOD Record 33(1), 32-37 (2004) 
24. Selim, S., Ismail, M.: Soft clustering of multidimensional data: a semi-fuzzy approach. Pattern Recognition 17(5), 559-568 (1984)

25. Tasoulis, D.K., Adams, N.M., Hand, D.J.: Unsupervised clustering in streaming data. In: ICDMW 2006: Proceedings of the Sixth IEEE International Conference on Data Mining - Workshops, pp. 638-642. IEEE Computer Society Press, Los Alamitos (2006)

26. Tu, L., Chen, Y.: Stream data clustering based on grid density and attraction. ACM Transactions Knowledge Discovery Data 3(3), 1-27 (2009)

27. Wan, L., Ng, W.K., Dang, X.H., Yu, P.S., Zhang, K.: Density-based clustering of data streams at multiple resolutions. ACM Transactions Knowledge Discovery Data 3(3), 1-28 (2009)

28. Xie, X., Beni, G.: A validity measure for fuzzy clustering. IEEE Transactions on pattern analysis and machine intelligence 13(8), 841-847 (1991)

29. Yang, C., Zhou, J.: Hclustream: A novel approach for clustering evolving heterogeneous data stream. In: ICDMW 2006: Proceedings of the Sixth IEEE International Conference on Data Mining - Workshops, pp. 682-688. IEEE Computer Society, Los Alamitos (2006)

30. Yang, J.: Dynamic clustering of evolving streams with a single pass. In: Proceedings of 19th International Conference on Data Engineering, March 2003, pp. 695-697 (2003)

31. Zhang, T., Ramakrishnan, R., Livny, M.: Birch: an efficient data clustering method for very large databases. SIGMOD Record 25(2), 103-114 (1996) 